This item was submitted to Loughborough's Research Repository by the author.

Items in Figshare are protected by copyright, with all rights reserved, unless otherwise indicated.

\title{
Thermal dynamics of silver clusters grown on rippled silica surfaces
}

PLEASE CITE THE PUBLISHED VERSION

http://dx.doi.org/10.1016/j.nimb.2016.10.005

\section{PUBLISHER}

(c) Elsevier

\section{VERSION}

AM (Accepted Manuscript)

\section{PUBLISHER STATEMENT}

This work is made available according to the conditions of the Creative Commons Attribution-NonCommercialNoDerivatives 4.0 International (CC BY-NC-ND 4.0) licence. Full details of this licence are available at: https://creativecommons.org/licenses/by-nc-nd/4.0/

\section{LICENCE}

CC BY-NC-ND 4.0

\section{REPOSITORY RECORD}

Bhatnagar, Mukul, Mukesh Ranjan, Kenny Jolley, Adam L. Lloyd, Roger Smith, and Subroto Mukherjee. 2019. "Thermal Dynamics of Silver Clusters Grown on Rippled Silica Surfaces". figshare.

https://hdl.handle.net/2134/24017. 


\title{
Thermal dynamics of silver clusters grown on rippled silica surfaces
}

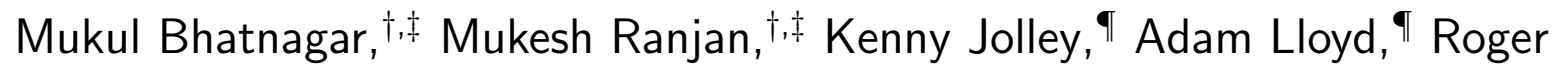 \\ Smith, and Subroto Mukherjee $e^{\dagger, \ddagger}$ \\ $\dagger$ †CIPT, Institute for Plasma Research, Gandhinagar, Gujarat, India \\ $\ddagger$ Nirma University, Ahmedabad, Gujarat, India \\ IDept. of Mathematical Sciences, Loughborough University, Leicestershire, LE11 3TU, UK
}

\section{Abstract}

Silver nanoparticles have been deposited on silicon rippled patterned templates at an angle of incidence of $70^{\circ}$ to the surface normal. The templates are produced by oblique incidence argon ion bombardment and as the fluence increases, the periods and heights of the structures increase. Structures with periods of 20 $\mathrm{nm}, 35 \mathrm{~nm}$ and $45 \mathrm{~nm}$ have been produced. Moderate temperature vacuum annealing shows the phenomenon of cluster coalescence following the contour of the more exposed faces of the ripple for the case of $35 \mathrm{~nm}$ and $45 \mathrm{~nm}$ but not at $20 \mathrm{~nm}$ where the silver aggregates into larger randomly distributed clusters. In order to understand this effect, the morphological changes of silver nanoparticles deposited on an asymmetric rippled silica surface are investigated through the use of Molecular Dynamics simulations for different deposition angles of incidence between $0^{\circ}$ and $70^{\circ}$ and annealing temperatures between $500 \mathrm{~K}$ and $900 \mathrm{~K}$. Near to normal incidence, clusters are observed to migrate over the entire surface but for deposition at $70^{\circ}$, a similar patterning is observed as in the experiment. The random distribution of clusters for the periodicity $\approx$ of $20 \mathrm{~nm}$ is linked to the geometry of the silica surface which has a lower ripple height than the longer wavelength structures. Calculations carried out on a surface with such a lower ripple height also demonstrate a similar effect.

\section{Introduction}

The wide tunability of Localised Surface Plasmon Resonance (LSPR) ${ }^{1,2}$ shown by silver nanoparticles (np) in the visible and near infrared spectrum has made them very useful in diverse applications including thermal applications $^{3}$, photovoltaics ${ }^{4,5}$ and bio nanoscience ${ }^{6}$. LSPR based research has seen a boom at the present time which is primarily due to the ease of fabrication of metallic nanoparticles in a variety of environments ${ }^{7,8}$. Especially, the case of ordered chains of silver nanoparticles on patterned templates ${ }^{9,10}$ is interesting for their use in nanoplasmonic waveguides ${ }^{11,12}$.

There have been a number of different studies of cluster growth on metal surfaces which combine experimental and modelling investigations. References ${ }^{13}$ and ${ }^{14}$ consider the ripening process in detail after the deposition of individual atoms whereas reference ${ }^{15}$ investigates the impact of clusters themselves onto a surface. Only few studies have focussed to understand the atomic interaction of silver with silica $^{16-18}$. In a previous paper ${ }^{19}$ we parametrised an interatomic potential function for the $\mathrm{Ag}-\mathrm{SiO}_{2}$ interaction, based on matching sticking probabilities to Rutherford backscattering data. Although a few research groups have studied the temperature dependent interaction of silver thin films and silver cluster with silica $^{20,21}$, there has been no investigation to understand the thermal dynamics of silver clusters 
deposited on nanopatterned templates.

Recently, a study based on the Kinetic Monte Carlo (KMC) method was undertaken to understand the mechanisms of the growth of silver nanoparticles on the patterned surfaces ${ }^{17}$. The reported study has limitations in some of the assumptions of the model such as no reflection of the Ag atom from the surface and no cluster migration on the surface. In addition, the energy and the angle of incidence (AOI) of the incoming atom were not parameters of the model ${ }^{17}$. In the present work, we have performed ex-situ SEM characterisation of the silver nanoparticles which shows patterned growth on rippled patterned templates of varying periodicity before and after annealing. We have used MD to understand the coalescence behaviour of the silver clusters deposited at different angles of incidence on the simulated rippled silica surface at $300 \mathrm{~K}$ and annealed at temperatures from $500 \mathrm{~K}$ to $900 \mathrm{~K}$. The simulated dynamical behaviour aids in the understanding of the experimentally observed patterns of silver np deposited at an angle of incidence of $70^{\circ}$, which occurs for the ripple wavelength $35 \mathrm{~nm}$ and above.

\section{Experimental Section}

The modification of a silicon surface to a rippled structure is a well-studied topic ${ }^{22,23}$. We report in brief, the fabrication process of silver nanoparticles on the rippled silica surface. An epipolised silicon wafer (100) was bombarded by a $500 \mathrm{eV}$ broad beam of $\mathrm{Ar}^{+}$ions at an AOI of $67^{\circ}$ with respect to the surface normal. The fluence was varied from $10^{16}-10^{19}$ ions $/ \mathrm{cm}^{2}$ to generate rippled templates with periodicities of 20 $\mathrm{nm}, 35 \mathrm{~nm}$ and $45 \mathrm{~nm}$. This generated rippled silicon structures with heights $\approx 1-2 \mathrm{~nm}$ for a wavelength of $20 \mathrm{~nm}, 2-3 \mathrm{~nm}$ for $35 \mathrm{~nm}$ and slightly higher at $45 \mathrm{~nm}$. The structure has an asymmetric nature where two planar surfaces lie at different angles with respect to the flat surface (see Fig. 1, inset in image 2). The substrates were then exposed to atmosphere which leads to the formation of a native oxide layer of approximate thickness $2 \mathrm{~nm}$ on the freshly bombarded surface. Subsequent deposition of silver was performed with a deposition rate of
$1 \mathrm{~nm} / \mathrm{sec}$ for 30 minutes at an AOI of $70^{\circ}$ using an electron beam evaporation system. The time duration of the deposition of silver was kept the same for the three periodic templates with pressure maintained at $10^{-8}$ mbar. The deposited samples were then annealed at $573 \mathrm{~K}$ for 1 hour and 4 hours, respectively. The underlying ripple pattern was not observed to change even at temperatures of upto $1000 \mathrm{~K}$.

\section{Results}

The top row of figure 1 shows the deposited silver on the rippled silica surface. Silver grows through a Volmer Weber mode that leads to the formation of separate clusters until the thickness of the film reaches several nanometres ${ }^{24}$. This feature of the growth is confirmed by the micrographs which show well separated np on the patterned template. It is seen by comparing images 1,2 and 3 that self-aligned silver np are obtained for a ripple periodicity greater than $20 \mathrm{~nm}$ whereas one observes less alignment at $20 \mathrm{~nm}$ and this disappears completely after annealing. Another feature visible from the images is that the $\mathrm{np}$ deposited on $\lambda=35 \mathrm{~nm}$ and above are well separated from np present on the neighbouring ripples as compared to $\lambda=20 \mathrm{~nm}$. This indicates that the onset of ordering starts at room temperature itself for these structures. The deposited silver np were then exposed to atmosphere and then annealed in vacuo at 573 $\mathrm{K}$ for a period of 1 hour as this leads to an approximately circular cross-section of $\mathrm{np}$ on account surface energy minimisation ${ }^{14,25}$. This is confirmed by the micrographs $\mathrm{A}, \mathrm{B}$ and $\mathrm{C}$ which show the np morphology of the annealed samples for $\lambda=20 \mathrm{~nm}, 35 \mathrm{~nm}$ and $45 \mathrm{~nm}$, respectively.

A common observation from all the images is the increase in the size of the silver np and evolution of the shape to a spherical one which is due to coalescence. Images $\mathrm{B}$ and $\mathrm{C}$ show that a configuration of ordered np chains is obtained for $\lambda=35 \mathrm{~nm}$ and $\lambda=45 \mathrm{~nm}$ whereas it is clearly visible that for $\lambda=20 \mathrm{~nm}$ silver $\mathrm{np}$ are randomly distributed similar to the flat substrate. The inset in image $\mathrm{C}$ shows the $\mathrm{np}$ morphology for the case where sample 3 was an- 

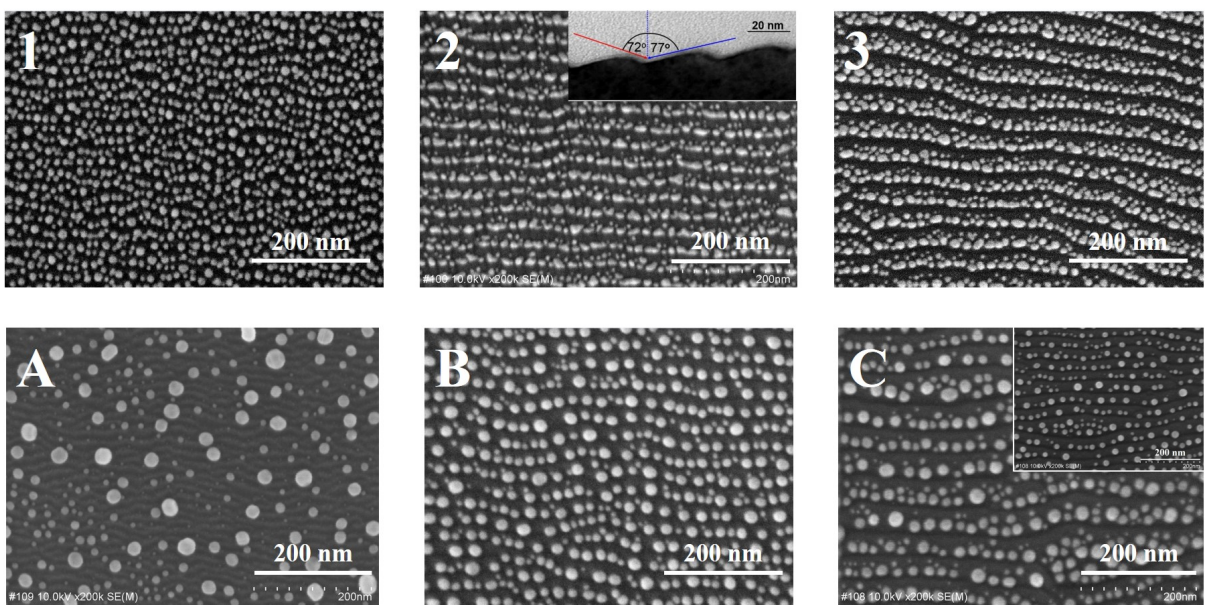

Figure 1: Scanning electron micrographs (SEM) showing the deposited silver nanoparticles on rippled silica surfaces. Images 1, 2 and 3 show the silver nanoparticle morphology on the rippled template with periodicity $\lambda=20 \mathrm{~nm}, 35 \mathrm{~nm}$ and $45 \mathrm{~nm}$, respectively. The inset in image 2 shows a cross sectional transmission electron micrograph of a $35 \mathrm{~nm}$ rippled structure. The asymmetry of the structure is clearly visible with the angle of inclination marked for each plane of the surface. Images $\mathrm{A}, \mathrm{B}$ and $\mathrm{C}$ show the system configuration for the three periodicities after being annealed at $573 \mathrm{~K}$ for a time duration of one hour. The inset in image $\mathrm{C}$ shows the morphology after annealing for 4 hours.
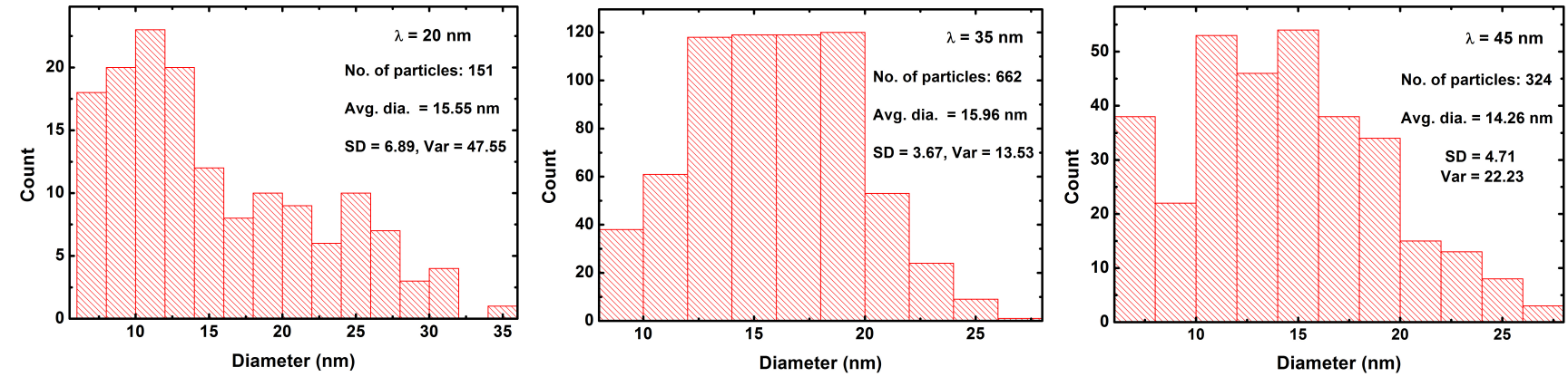

Figure 2: Histograms showing the number of particles and the measured average diameter for the annealed silver np for the three different ripple periodicities.

nealed for a time duration of 4 hours. It is seen from the image that the ordered configuration is still not lost and a clear features are observed with an ordering that follows the ripple pattern on the template. For the $20 \mathrm{~nm}$ wavelength case not only are the annealed np more randomly spread, they also have a larger average diameter than in the other cases (see Fig. 2). This indicates a more uniform deposition and isotropic coalescence which is attributed to the lower height of the structure for the smaller wavelength case. Figure 2 shows the histograms plotted from the micrographs for the samples annealed at $573 \mathrm{~K}$ for 1 hour. Although the variation in average size is around one $\mathrm{nm}$ for the three cases, the spread in the np size distribution is much more for $\lambda=20 \mathrm{~nm}$ as seen through the standard deviation (SD) and the variance (var) given in the figure. The maximum diameter of $\mathrm{np}$ for $\lambda=20 \mathrm{~nm}$ is measured to be $34.6 \mathrm{~nm}$ whereas it is $27.8 \mathrm{~nm}$ and $26.9 \mathrm{~nm}$ for $\lambda=35 \mathrm{~nm}$ and 45 $\mathrm{nm}$, respectively. To understand these effects, we performed $\mathrm{MD}$ simulation of the $\mathrm{Ag}-\mathrm{SiO}_{2}$ system and discuss the results in the following section.

\section{Molecular Dynamics Simulations}

We simulated the interaction between silver adatoms and a rippled silica template using mo- 
lecular dynamics (MD). MD simulations are limited to time scales up to microseconds at best and experimental time scales are generally not accessible by MD alone. Recently a multi-time scale technique has been developed that uses MD to model individual particle impacts over picosecond time scales followed by an off-lattice KMC approach to model diffusion between particle impacts ${ }^{26}$. This works well when the diffusion barriers are large so that atom movement only occurs as series of rare events. If the energy barriers for diffusion are low then the time acceleration is much more modest. We performed some simulations using this off-lattice KMC technique but found that the computed barriers were low and that $\mathrm{Ag}$ atoms diffused quickly to form clusters on the surface. The barriers for a single Ag to diffuse from one local minimum to another, calculated using the nudged elastic band technique ${ }^{27}$, varied between 0.1 and $0.2 \mathrm{eV}$ depending on the attachment site. Small clusters containing up to $4 \mathrm{Ag}$ atoms were also found to be mobile on the surface over picosecond time scales at room temperature. Once the larger Ag clusters were formed it was difficult for them to break apart although in some cases during a deposition event, an entire cluster could detach from the surface due to the weak bonding with the substrate. Even the deposition of a few atoms using the off-lattice KMC involved weeks of computing time. As a result we performed MD simulations at unrealistically high deposition rates for comparison. It was found that the results were qualitatively the same as using off-lattice KMC.

In a MD simulation, all material properties such as bond angles, bond lengths, coordination numbers, bulk modulus etc. are defined within the interatomic potential ${ }^{28}$. A two body fixed charge potential developed by Kieu et al. containing a Coloumb term and a dipole term was used to simulate the $\mathrm{SiO}_{2}$ substrate ${ }^{29}$. We used a Morse potential to simulate the silver silica interaction. The potential was chosen to be consistent with reflection coefficients calculations and with results from Rutherford backscattering experiments ${ }^{19}$. The equation for Morse po- tential is given as;

$$
\begin{aligned}
V(r)= & \frac{D_{e}}{S-1}\left\{\exp \left[-(2 S)^{\frac{1}{2}} \beta\left(r-r_{e}\right)\right]\right\} \\
& -S \exp \left\{\left[-(2 / S)^{\frac{1}{2}} \beta\left(r-r_{e}\right)\right]\right\}
\end{aligned}
$$

where $r_{e}$ is the equilibrium bond length, $D_{e}$ is the potential energy well depth at $r_{e}, \beta$ and $S$ are free parameters which take the value 2 in the standard Morse potential. The first term on the right hand side simulates the short range repulsion whereas the latter term accounts for the long range attraction ${ }^{28}$. Tables I shows the value of these parameters for average binding energy value of $0.2 \mathrm{eV}$ for an Ag atom on the surface.

Table 1: Parameters of Morse potential for average $E_{b}=0.2 \mathrm{eV}$

\begin{tabular}{ccccc}
\hline \hline Bond & $D_{e}(\mathrm{eV})$ & $r_{e}(\stackrel{\circ}{ })$ & $\beta\left(A^{-1}\right)$ & $S$ \\
\hline $\mathrm{Ag}-\mathrm{Si}$ & 0.05 & 2.6 & 3.0 & 2.0 \\
$\mathrm{Ag}-\mathrm{O}$ & 0.05 & 2.6 & 3.0 & 2.0 \\
\hline \hline
\end{tabular}

The Ag binding energy is defined as $E_{b}=$ $E_{\mathrm{Ag}-\mathrm{SiO}_{2}}-E_{\mathrm{SiO}_{2}}$. Here $E_{\mathrm{Ag}-\mathrm{SiO}_{2}}$ is the potential energy of the system with a silver atom present on the silica surface and $E_{\mathrm{SiO}_{2}}$ is the potential energy of the silica substrate. The Ackland potential was used to simulate the $\mathrm{Ag}-\mathrm{Ag}$ interaction ${ }^{30}$. All the potentials used in this work are splined to the ZBL potential through a sixth order polynomial to describe the nucleus-nucleus repulsion at very small distances $^{31}$. However this part of the energetic interaction does not come into play for the low energies involved in the simulations presented here.

We report in brief, the setup used to generate the rippled silica substrate (R1). The dimensions of the simulation box was calculated to hold 999 atoms $\left(\mathrm{N}_{\mathrm{Si}}=333\right.$ and $\left.\mathrm{N}_{\mathrm{O}}=666\right)$ with a density $2.65 \mathrm{~g} / \mathrm{cm}^{3}$ with periodic boundary conditions in all directions. This gave the Cartesian dimensions of the bounding box as $\mathrm{X}=2.92 \mathrm{~nm}, \mathrm{Y}=1.46 \mathrm{~nm}$ and $\mathrm{Z}=2.92$ $\mathrm{nm}$. The system was then quenched as reported elsewhere ${ }^{29}$. Periodic copies of the system 

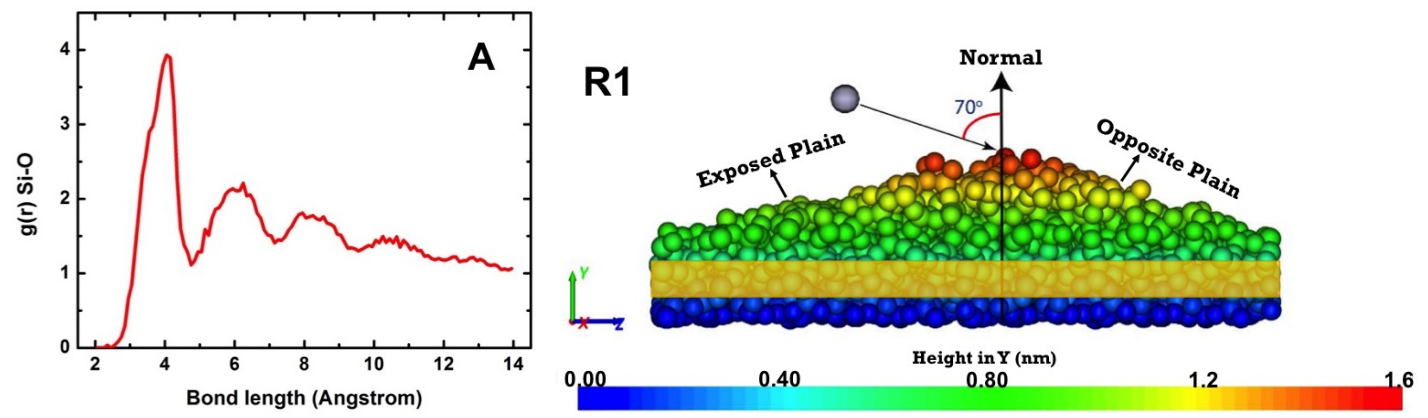

Figure 3: (Colour online) A: Plot shows the rdf for the Si-O atomic pair. The image on the right shows a profile for a cross deposition event at an AOI $70^{\circ}$. The topmost atom of the surface is at a height of $1.56 \mathrm{~nm}$ above the bottom. The translucent yellow bar marks the region of the substrate $(\approx 2-3$ atomic layers) which was coupled to the thermostat during the time period of the simulation.

were made so that the two edge lengths of the quenched substrate in the $\mathrm{Y}$ and the $\mathrm{Z}$ direction became $\mathrm{Y}=2.92 \mathrm{~nm}$ and $\mathrm{Z}=5.84 \mathrm{~nm}$. This substrate was then cut at angles consistent with those shown in the TEM image (Fig. 2B) to produce a surface with average surface normal in the $\mathrm{Y}$ direction. This leads to a triangulated structure where the top most atom of the silica substrate has a height of $1.56 \mathrm{~nm}$. The empty space from $\mathrm{Y}=1.56 \mathrm{~nm}$ to 2.92 $\mathrm{nm}$ within the simulation box serves as vacuum above the surface of the substrate. The bottom layer of the system was fixed and the substrate was then relaxed using damped $\mathrm{MD}^{32}$ which did not cause an appreciable change in the angles of inclination of the two planes. The system was finally thermalised to $300 \mathrm{~K}$ using the Berendsen thermostat ${ }^{33}$ before the start of the production run. The system set up is shown in Fig. 3. During the production run, the system was kept coupled to the thermostat so as to remove excess energy due to the accumulation of silver adatoms. Some final configurations were then annealed at different temperatures. Figure 3 shows the radial distribution function (rdf) for Si-O atomic pair. The rdf has broadened peaks and goes to 1 as the distance increases which should be the case for an amorphous or glassy substrate. Similar plots were obtained for other atomic pairs of the substrate. The image on the right shows the side view of the silica substrate with both planes of the rippled surface explicitly marked. The normal of the exposed plane lies in the direction of the incoming silver atoms while that of the opposite plane points away.

In a typical electron beam evaporation experiment, the kinetic energy of the evaporated atoms from the crucible containing the material has an energy much less than $1.0 \mathrm{eV}^{34,35}$ with a narrow energy distribution ${ }^{36}$. Therefore, we fixed $E_{A g}=0.1 \mathrm{eV}$ as the kinetic energy for the $\mathrm{Ag}$ adatom. The bottom 3 layers of the substrate were fixed and periodic boundary conditions were applied in the $\mathrm{X}$ and $\mathrm{Z}$ directions of the substrate. Each simulation was continued until the system equilibrated or the $\mathrm{Ag}$ atom was found to evaporate from the surface and reach a distance of more than $0.5 \mathrm{~nm}$ from the topmost atom of the surface (the interaction range for $\mathrm{Ag}-\mathrm{Ag}=0.5 \mathrm{~nm}, \mathrm{Ag}-\mathrm{SiO}_{2}=0.35$ $\mathrm{nm})$. The equilibration time was around $20 \mathrm{ps}$ in all cases after which the substrate temperature settled down to the heat bath level and the next $\mathrm{Ag}$ atom was deposited. Cumulative depositions were performed at AOI $=0^{\circ}, 20^{\circ}$, $40^{\circ}, 60^{\circ}$ and $70^{\circ}$ with the substrate coupled to the thermostat fixed at a temperature of 300 K. A total of 132 deposition events were simulated for each angle of incidence. Figure 4 shows the final snapshots for these angles of incidence. The strong ionic nature of the substrate did not result in any broken $\mathrm{Si}-\mathrm{O}$ bonds and substrate atoms only oscillated around their initial positions after a deposition event. The results from the cumulative depositions clearly show that the angle of incidence plays a crucial role in the formation of silver clusters on the rippled surface. The effect of angle can be understood 

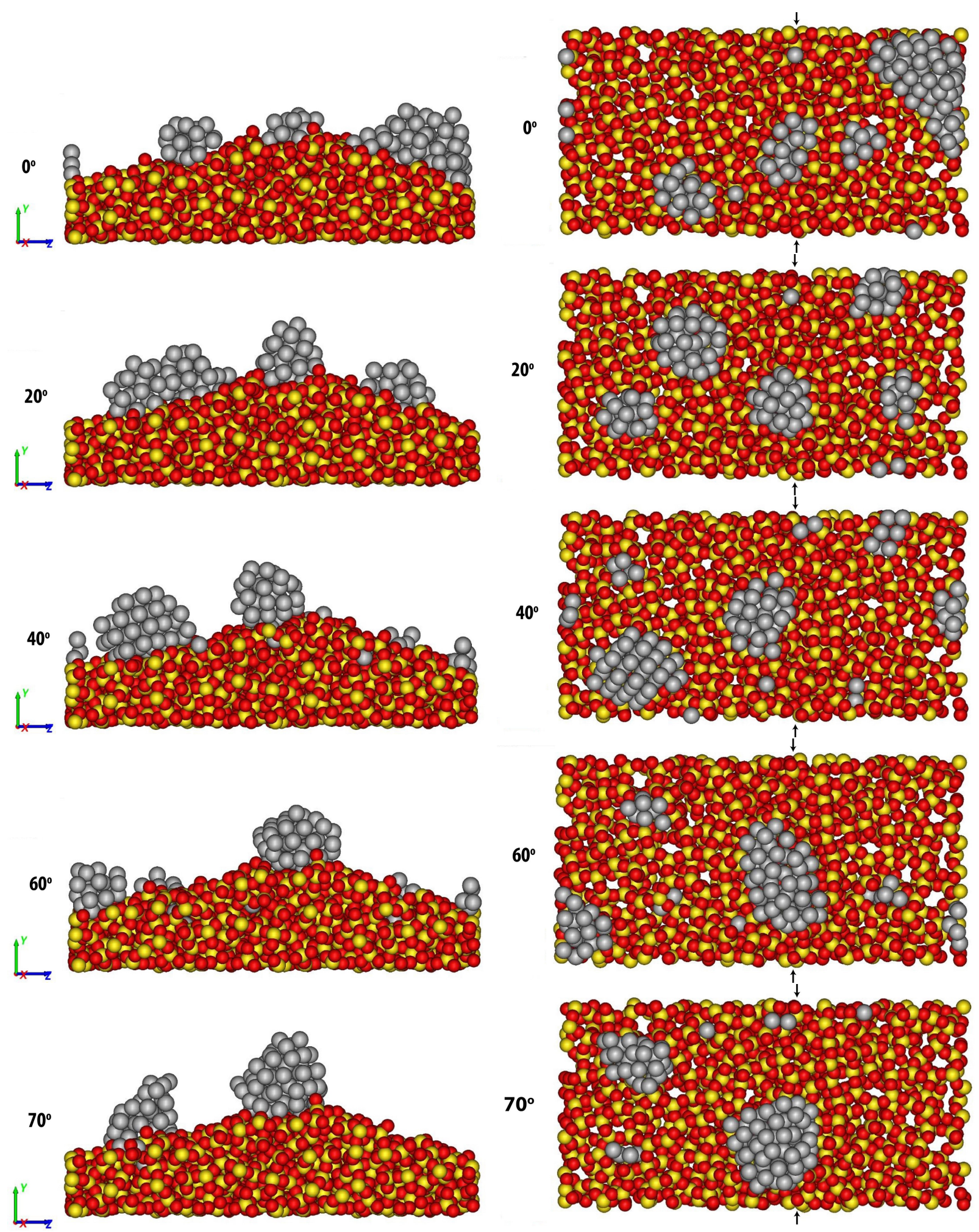

Figure 4: Final snapshots from the cumulative deposition of silver on the rippled silica surface at different AOI. The left column shows the side view whereas the right column shows the corresponding top view. It is seen that as the AOI increases from the surface normal, the number of silver atoms present on the more exposed plane is greater than the opposite plane of the rippled surface. Here $E_{A g}=0.1 \mathrm{eV}$ and $\mathrm{T}=300 \mathrm{~K}$. 
in terms of the apparent area of the surface visible to the incoming adatom. We discuss the effect for the case of $0^{\circ}$ and $70^{\circ}$.

Due to the asymmetric nature of the rippled surface, the angle made by the vector of the $\mathrm{Ag}$ adatom (incident at $70^{\circ}$ w.r.t the surface normal) with the exposed plane is $57^{\circ}$ while that with the opposite plane is $88^{\circ}$. Previously ${ }^{19}$ the variation of the sticking probability with incidence angle was determined. For an $\mathrm{AOI}=70^{\circ}$ the silver clusters stick preferentially on the exposed plane with a reduced sticking probability on the other plane whereas one does not observe much difference for the AOI $0^{\circ}$ case. The observed growth of the clusters is in fair agreement with the current experimental results as well as with the earlier reported investigations on the observed self-assembly of silver np for AOI $70^{\circ} 37$.

To study the temperature dependent behaviour of the deposited clusters, we heat treated all the systems with final temperatures from $\mathrm{T}$

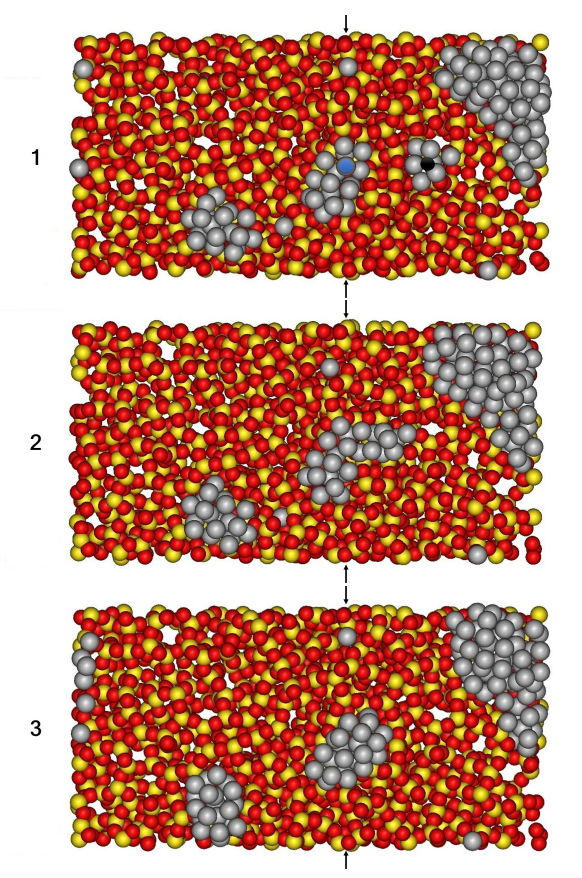

Figure 5: Snapshots showing the migration of silver clusters on the rippled silica surface for an $\mathrm{AOI}=0^{\circ}$ at $\mathrm{T}=600 \mathrm{~K}$. The cluster present on the ridge of the rippled surface is marked blue (A) while that present on the slope is marked black (B). The two clusters merge together within a time scale of tens of picoseconds.
$=500 \mathrm{~K}$ to $\mathrm{T}=900 \mathrm{~K}$ in steps of $100 \mathrm{~K}$. For all the simulation runs, the system was coupled to the thermostat to maintain the system at the desired temperature. The length of the simulation was 1 nanosecond (ns). A movie file was created for all simulation runs to understand the dynamical behaviour of the clusters. As the temperature was increased to $900 \mathrm{~K}$, concerted motions by clusters take place on the silica surface. This also occurs at lower temperatures but over much longer time scales. This behaviour confirms that the silver clusters form through cluster migration on the surface and not just by adatom attachment as was assumed in the previous KMC model. Other studies have also confirmed the observed coalescence behaviour $^{38}$.

Fig. 5 shows a cluster coalescence event observed for an AOI $=0^{\circ}$. Image 1 shows the cluster configuration after 132 deposition events. After 12 picoseconds (image 2), it is seen that the cluster $B$ joins the cluster on the ridge and at $t=24$ ps (image 3 ) we only see a single cluster present on the top portion of the rippled surface. Although the two small clusters merge together, the nearby isolated adatom has only moved 1 step. This is because diffusion on an amorphous surface is site dependent, the value of $E_{b}=0.2 \mathrm{eV}$ being only an average value with some adatoms being more strongly bound than others. With the formation of a close-packed Ag structure, the height of the cluster also increases which is in agreement with the experimental results ${ }^{39}$.

These simulations also shed light on the observed ordered coalescence of the silver np (see Fig. 1) which is not observed when silver np are deposited and annealed on a flat silica surface. This property of silver np to form an ordered chain on the rippled surface is particularly favourable when silver is deposited at an $\mathrm{AOI}=$ $70^{\circ}$. To gain insight to the observed coalescence of silver np, we searched for clusters that performed a crossing over the ridge made by the asymmetric tilt of the two planes. Table II shows this behaviour of the deposited clusters for different angles of incidence. The cluster mobility over the ridge was found to be negligible at $500 \mathrm{~K}$. For $600 \mathrm{~K}$ onwards, it was ob- 
Table 2: Observed crossover of the silver clusters on the rippled surface for different angles of incidence at different temperatures. $X$ - cluster migration across the ridge does not occur. $\checkmark$ cluster migration present across the ridge. In all the cases, $E_{A g}=0.1 \mathrm{eV}$ with the number of deposition events $=132$

\begin{tabular}{cccccc}
\hline \hline AOI \Temp. & $500 \mathrm{~K}$ & $600 \mathrm{~K}$ & $700 \mathrm{~K}$ & $800 \mathrm{~K}$ & $900 \mathrm{~K}$ \\
$0^{\circ}$ & $X$ & $\checkmark$ & $\checkmark$ & $\checkmark$ & $\checkmark$ \\
$20^{\circ}$ & $X$ & $X$ & $X$ & $\checkmark$ & $X$ \\
$40^{\circ}$ & $X$ & $\checkmark$ & $\checkmark$ & $\checkmark$ & $\checkmark$ \\
$60^{\circ}$ & $X$ & $X$ & $\checkmark$ & $\checkmark$ & $\checkmark$ \\
$70^{\circ}$ & $X$ & $X$ & $X$ & $X$ & $\checkmark$ \\
\hline \hline
\end{tabular}
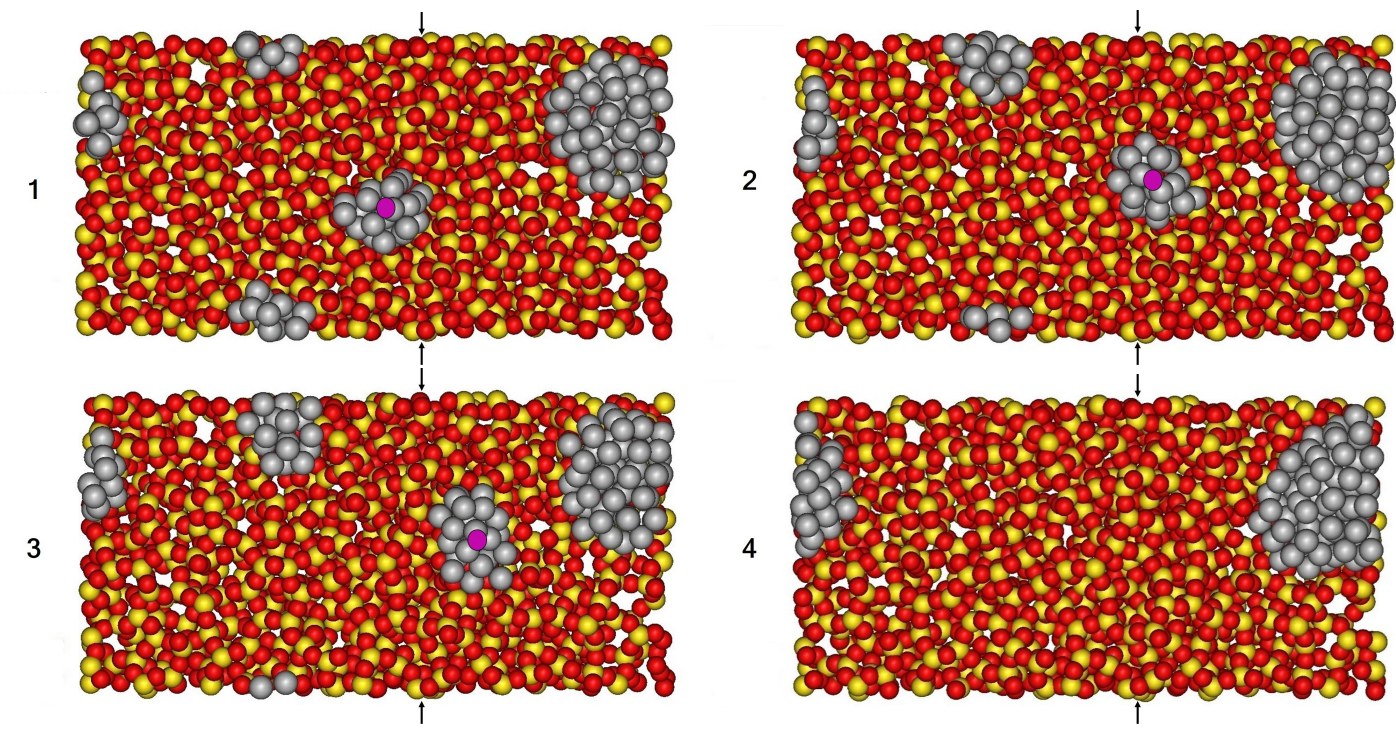

Figure 6: Image 1-3: Snapshots showing the migration of the silver cluster (purple) over the ridge portion of the rippled silica surface deposited with an $\mathrm{AOI}=0^{\circ}$ and $\mathrm{T}=600 \mathrm{~K}$. Image 4 shows the system configuration at time $\mathrm{t}=1 \mathrm{~ns}$.

served that the crossover was present for AOI $0^{\circ}$. As an example, we show in figure 6 the snapshots of crossover of a silver cluster from one plane to the other.

The purple marked silver cluster moves from one plane to the other within a span of 18 ps and merges into the larger cluster present on the corner of the surface during the time scale of the simulation. Thus in our case, there are three key parameters which play an important role to understand the observed motion of silver clusters; 1.) the site where the cluster is bonded to the surface; a deeper potential well causes reduced cluster mobility. 2.) the size of the cluster; bigger the cluster, the lesser the mo- bility. 3.) the temperature to which the system is heat treated; a higher temperature leads to increased mobility along with the evaporation from the surface.

It was observed from figure 1 that silver $n p$ deposited at $\mathrm{AOI}=70^{\circ}(\lambda \approx 35 \mathrm{~nm})$ show an ordered coalescence when annealed at a temperature of $573 \mathrm{~K}$. This behaviour is also observed through the MD simulations of heat treatment of the silver clusters deposited at an $\mathrm{AOI}=70^{\circ}$ (see table II). Fig 7. gives an example of the dynamical behaviour of the silver clusters when heat treated at $600 \mathrm{~K}$.

It is seen from images 1,2 and 3 in figure 7 that the two clusters coalesce (at time $\mathrm{t}=$ 


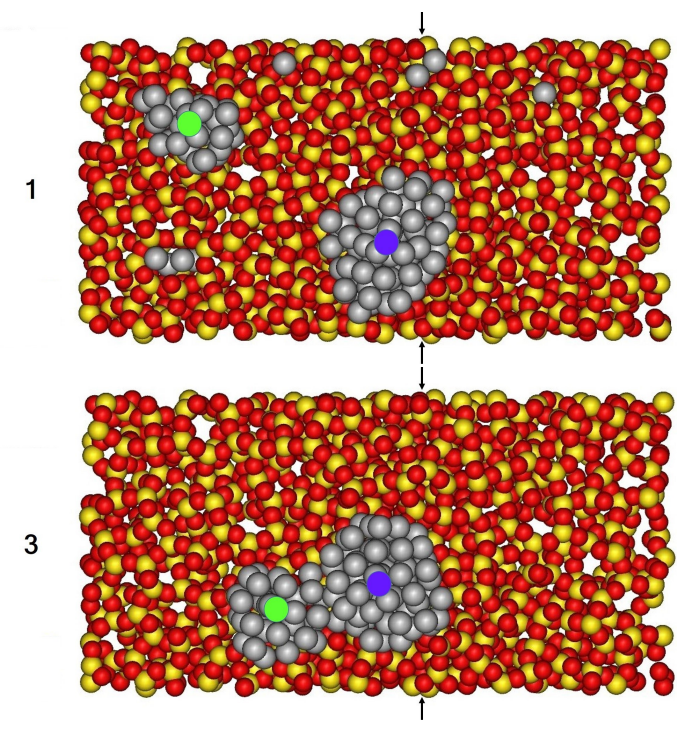

2

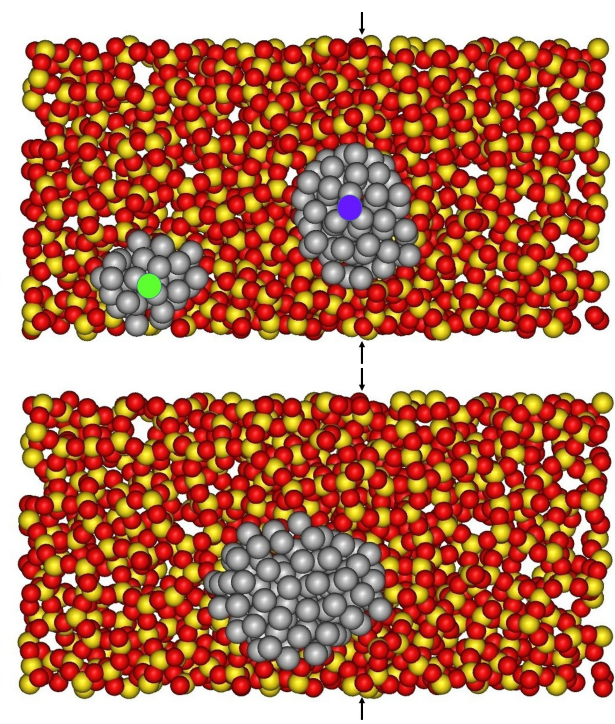

Figure 7: Snapshots showing the coalescence of the silver clusters at specified time for $\mathrm{T}=600 \mathrm{~K}$. The two clusters are marked as green and blue. The deposition parameters are $E_{A g}=0.1 \mathrm{eV}$, AOI $=70^{\circ}$ and $\mathrm{T}=300 \mathrm{~K}$.

$574 \mathrm{ps)}$ and remain on the exposed plane of the rippled surface until the end of the simulation. At an $\mathrm{AOI}=70^{\circ}$, the deposited clusters are present only on the exposed plane of the surface. When the temperature of the system is raised, the clusters, depending upon their size and the substrate site to which they are bonded, move on the surface which in turn increases the probability to interact with a nearby cluster, an event more favourable for the clusters present on the same plane of the rippled surface. This behaviour indicates towards the experimentally observed patterns of silver np, although our system suffers from the limitation of a lower periodicity $(\approx 5.84 \mathrm{~nm})$ than experiment.

An important observation from SEM images of annealed silver np on ripple structure with periodicity $\approx 20 \mathrm{~nm}$ (fig. 1, image $A$ ) is that the np are found to be present all over the template (no ordered pattern) as against the case for $\lambda=35 \mathrm{~nm}$ or $\lambda=45 \mathrm{~nm}$. To understand this experimental observation, we performed cumulative deposition of silver on a different rippled surface. The dimensions in $\mathrm{X}$ and $\mathrm{Z}$ directions were same as the previous rippled surface but the angle of inclination was reduced by $5^{\circ}$ for the two planes. This leads to an inclination of $8^{\circ}$ for the exposed plane and $13^{\circ}$ for the opposite plane from the surface horizontal. We name this surface R2. As before, the number of deposition events was fixed to 132 with a time interval of 20 ps between each deposition event. The AOI for deposition was $70^{\circ}$ with $E_{A g}=0.1 \mathrm{eV}$. Fig. 8 (image A and image B) shows the results from this simulation.

On comparing the simulation results of the cumulative deposition between the two rippled surfaces [R1(figure 4) v/s R2], it is seen that the silver clusters grow on the exposed plane only for R1 whereas clusters are present on the both planes for R2. This is due to the reduction in the angle of the inclination of the planes for R2 as this leads to apparent angles of $62^{\circ}$ and $83^{\circ}$ for the exposed plane and the opposite plane thereby leading to an increase in the sticking probability on the Ag atom on the opposite plane of the surface. The above result supports our experimental results (fig.1, image 1) which shows that the deposited silver np are present on the rippled structure $(\lambda=20 \mathrm{~nm})$ without any global alignment as observed for the other two periodicities.

We now compare the results from the heat treatment of the deposited clusters on the two surfaces. The deposited clusters on R2 were heat treated at $\mathrm{T}=600 \mathrm{~K}$ for a time period of 1 ns. The clusters deposited on R1 show a distinct patterns (fig. 7) whereas for the case of R2, snapshots 1, 2 and 3 (fig. 8) show that the cluster migration is present across the ridge 


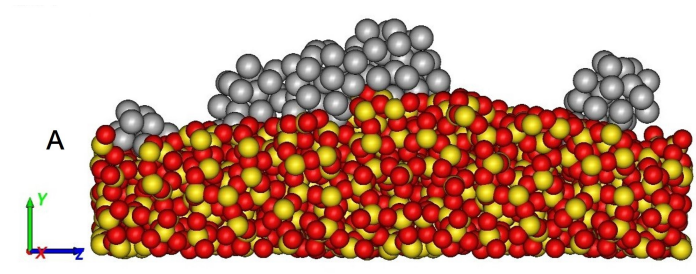

B

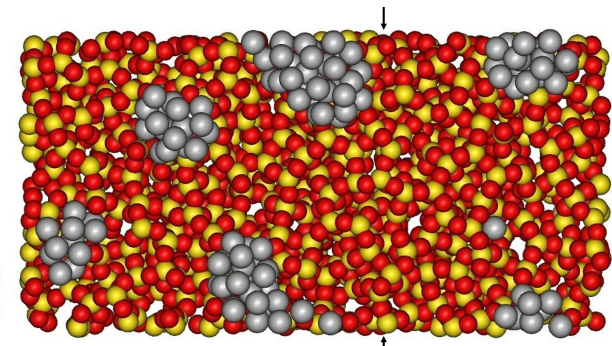

2
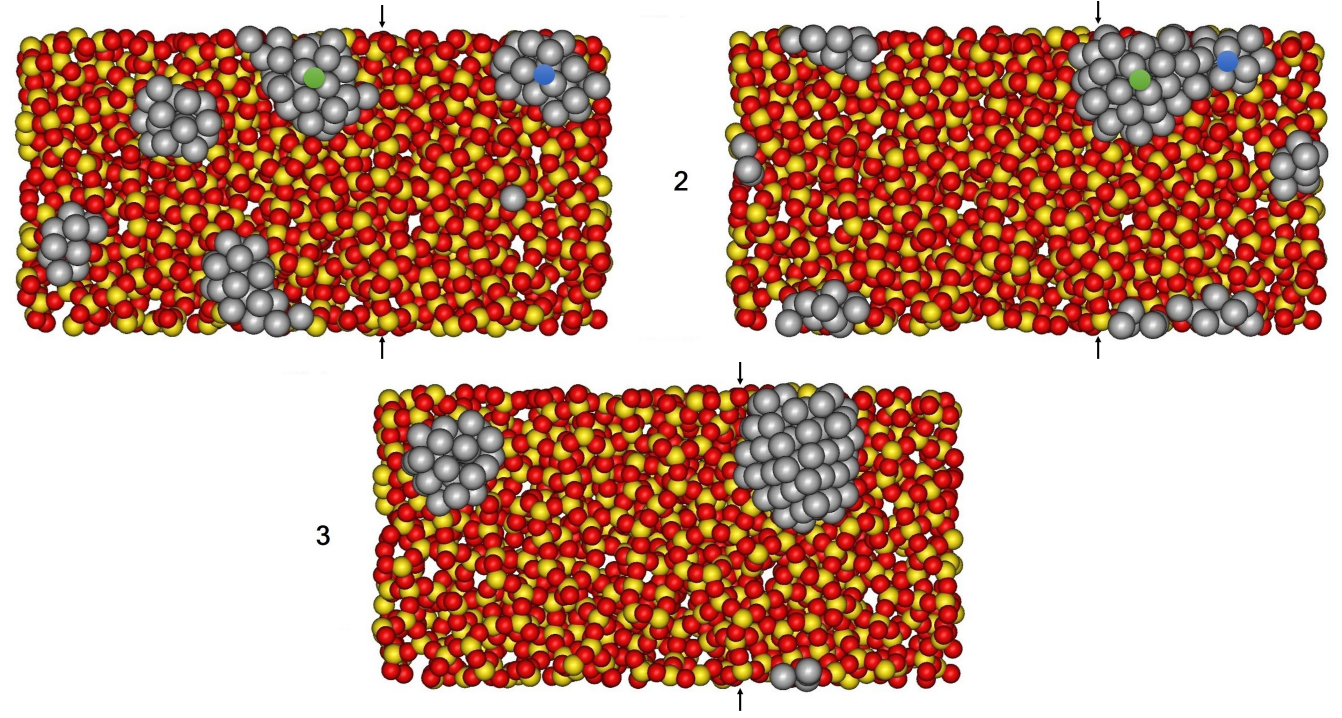

Figure 8: Images A and B show the side view and the top view of the deposited silver on the rippled surface. The total number of deposition events is $132, \mathrm{~T}=300 \mathrm{~K}, \mathrm{AOI}=70^{\circ}$. Images 1,2 and 3 show the snapshots at specific time intervals from the heat treatment simulation at $\mathrm{T}=600 \mathrm{~K}$. The two clusters marked green and blue are seen to coalesce with cluster migration from one plane to the other. Image 3 shows that only two clusters are present with no further coalescence until the end of the simulation.

formed by the two planes. In particular, the green marked cluster migrates from the exposed plane towards the blue marked cluster present on the opposite plane within a timescale of 300 ps. This behaviour was not observed for R1 as the opposite plane was devoid of clusters during the cumulative deposition. These observations are in good agreement with our experimental results for silver np deposited on a rippled template with $\lambda=20 \mathrm{~nm}$ which does not show the ordered patterning as compared to $\lambda=35 \mathrm{~nm}$ and $\lambda=45 \mathrm{~nm}$.

\section{Conclusion}

Self-alignment due to temperature induced coalescence is observed for silver np deposited on the rippled template with periodicity $35 \mathrm{~nm}$ and $45 \mathrm{~nm}$. For the ripple periodicity of 20 $\mathrm{nm}$, we do not observe self-alignment of silver np as the np are found to be distributed in a random manner over the substrate identical to the coalescence behaviour on a flat substrate. This feature is also reflected through the histograms for the measured size of the silver $\mathrm{np}$ which show a narrow distribution for $\lambda=35$ $\mathrm{nm}$ and $\lambda=45 \mathrm{~nm}$.

The results from MD simulations of the cumulative deposition of silver on the rippled surface show that the angle of incidence plays a crucial role to obtain a configuration of self-aligned silver np. In particular, the observed growth of silver clusters for the AOI of $70^{\circ}$ takes place on the exposed plane of the rippled surface. The simulation results from the heat treatment of the deposited silver clusters show that cluster migration across the ridge of the rippled surface is present only for $\mathrm{AOI}<70^{\circ}$ in the temperature range $600 \mathrm{~K}$ to $800 \mathrm{~K}$ which is in agreement 
with the experimental observations.

Acknowledgement This work was supported by by the DST - British Council Exchanges with India scheme, UKIERI Grant: IND/CONT/E/13-14/642.

\section{References}

(1) Maier, S. A. Plasmonics: fundamentals and applications; Springer Science \& Business Media, 2007.

(2) Amendola, V.; Bakr, O. M.; Stellacci, F. A study of the surface plasmon resonance of silver nanoparticles by the discrete dipole approximation method: effect of shape, size, structure, and assembly. Plasmonics 2010, 5, 85-97.

(3) Link, S.; El-Sayed, M. A. Shape and size dependence of radiative, non-radiative and photothermal properties of gold nanocrystals. International Reviews in Physical Chemistry 2000, 19, 409-453.

(4) Paetzold, U. W.; Moulin, E.; Pieters, B. E.; Carius, R.; Rau, U. Design of nanostructured plasmonic back contacts for thin-film silicon solar cells. Optics express 2011, 19, A1219-A1230.

(5) Tan, H.; Sivec, L.; Yan, B.; Santbergen, R.; Zeman, M.; Smets, A. H. Improved light trapping in microcrystalline silicon solar cells by plasmonic back reflector with broad angular scattering and low parasitic absorption. Applied Physics Letters 2013, 102, 153902.

(6) Miao, P.; Liu, T.; Li, X.; Ning, L.; Yin, J.; Han, K. Highly sensitive, label-free colorimetric assay of trypsin using silver nanoparticles. Biosensors and Bioelectronics 2013, 49, 20-24.

(7) Starowicz, Z.; Lipiński, M.; Socha, R.; Berent, K.; Kulesza, G.; Ozga, P. Photochemical silver nanoparticles deposition on sol-gel $\mathrm{TiO} 2$ for plasmonic properties utilization. Journal of Sol-Gel Science and Technology 2015, 73, 563-571.

(8) Daeneke, T.; Carey, B.; Chrimes, A.; Ou, J. Z.; Lau, D.; Gibson, B.; Bhaskaran, M.; Kalantar-Zadeh, K. Light driven growth of silver nanoplatelets on 2D MoS 2 nanosheet templates. Journal of Materials Chemistry C 2015, 3, 47714778 .

(9) Camelio, S.; Vandenhecke, E.; Rousselet, S.; Babonneau, D. Optimization of growth and ordering of Ag nanoparticle arrays on ripple patterned alumina surfaces for strong plasmonic coupling. $\mathrm{Na}$ notechnology 2013, 25, 035706.

(10) Ranjan, M.; Bhatnagar, M.; Mukherjee, S. Localized surface plasmon resonance anisotropy in template aligned silver nanoparticles: A case of biaxial metal optics. Journal of Applied Physics 2015, 117, 103106.

(11) Fang, Y.; Sun, M. Nanoplasmonic waveguides: towards applications in integrated nanophotonic circuits. Light: Science $\&$ Applications 2015, 4, e294.

(12) Ross, M. B.; Mirkin, C. A.; Schatz, G. C. The Optical Properties of One-, Two-, and Three-Dimensional Arrays of Plasmonic Nanostructures. The Journal of Physical Chemistry $C$ 2015,

(13) Brune, H. Microscopic view of epitaxial metal growth: nucleation and aggregation. Surface Science Reports 1998, 31, 125-229.

(14) Jensen, P. Growth of nanostructures by cluster deposition: Experiments and simple models. Reviews of Modern Physics 1999, 71, 1695.

(15) Popok, V. N.; Barke, I.; Campbell, E. E.; Meiwes-Broer, K.-H. Cluster-surface interaction: From soft landing to implantation. Surface Science Reports 2011, 66, 347-377. 
(16) Galashev, A.; Polukhin, V. Computerassisted study of silver absorption by porous silicon dioxide nanoparticles. Colloid journal 2011, 73, 761-767.

(17) Numazawa, S.; Ranjan, M.; Heinig, K.-H.; Facsko, S.; Smith, R. Ordered Ag nanocluster structures by vapor deposition on pre-patterned SiO2. Journal of Physics: Condensed Matter 2011, 23, 222203.

(18) Numazawa, S.; Smith, R. Markov-chain model of classified atomistic transition states for discrete kinetic Monte Carlo simulations. Physical Review E 2011, 84, 046714.

(19) Bhatnagar, M.; Ranjan, M.; Jolley, K.; Smith, R.; Mukherjee, S. Sub-monolayer growth of $\mathrm{Ag}$ on flat and nanorippled $\mathrm{SiO} 2$ surfaces. Applied Physics Letters 2016, 108.

(20) Wenzel, T.; Bosbach, J.; Stietz, F.; Träger, F. In situ determination of the shape of supported silver clusters during growth. Surface science 1999, 432, 257264.

(21) Kim, H.; Theodore, N.; Alford, T. Comparison of texture evolution in $\mathrm{Ag}$ and $\mathrm{Ag}$ $(\mathrm{Al})$ alloy thin films on amorphous $\mathrm{SiO} 2$. Journal of applied physics 2004, 95, 51805188.

(22) Keller, A.; Facsko, S.; Möller, W. Evolution of ion-induced ripple patterns on $\mathrm{SiO}$ 2 surfaces. Nuclear Instruments and Methods in Physics Research Section B: Beam Interactions with Materials and Atoms 2009, 267, 656-659.

(23) Ranjan, M.; Facsko, S.; Fritzsche, M.; Mukherjee, S. Plasmon resonance tuning in Ag nanoparticles arrays grown on ripple patterned templates. Microelectronic Engineering 2013, 102, 44-47.

(24) Lončarić, M.; Sancho-Parramon, J.; Pavlović, M.; Zorc, H.; Dubček, P.; Turković, A.; Bernstorff, S.; Jakopic, G.;
Haase, A. Optical and structural characterization of silver islands films on glass substrates. Vacuum 2009, 84, 188-192.

(25) Lehtinen, K. E.; Zachariah, M. R. Effect of coalescence energy release on the temporal shape evolution of nanoparticles. Physical Review B 2001, 63, 205402.

(26) Scott, C.; Blackwell, S.; Vernon, L.; Kenny, S.; Walls, M.; Smith, R. Atomistic surface erosion and thin film growth modelled over realistic time scales. The Journal of chemical physics 2011, 135, 174706 .

(27) Henkelman, G.; Uberuaga, B. P.; Jónsson, H. A climbing image nudged elastic band method for finding saddle points and minimum energy paths. The Journal of chemical physics 2000, 113, 9901-9904.

(28) Smith, R. Atomic and ion collisions in solids and at surfaces: theory, simulation and applications; Cambridge University Press, 2005.

(29) Jolley, K.; Smith, R.; Joseph, K. Borosilicate glass potentials for radiation damage simulations. Nuclear Instruments and Methods in Physics Research Section B: Beam Interactions with Materials and Atoms 2015, 352, 140-144.

(30) Ackland, G.; Tichy, G.; Vitek, V.; Finnis, M. Simple N-body potentials for the noble metals and nickel. Philosophical Magazine A 1987, 56, 735-756.

(31) Ziegler, J. F.; Biersack, J. P. The stopping and range of ions in matter; Springer, 1985 .

(32) Lindhard, J.; Scharff, M.; Schiøtt, H. E. Range concepts and heavy ion ranges; Munksgaard, 1963.

(33) Berendsen, H. J.; Postma, J. v.; van Gunsteren, W. F.; DiNola, A.; Haak, J. Molecular dynamics with coupling to an external bath. The Journal of chemical physics 1984, 81, 3684-3690. 
(34) Asano, T.; Uetake, N.; Suzuki, K. Mean atomic velocities of uranium, titanium and copper during electron beam evaporation. Journal of Nuclear Science and Technology 1992, 29, 1194-1200.

(35) Groves, J. F. Directed vapor deposition. Ph.D. thesis, University of Virginia, 1998.

(36) Chopra, K. Thin Film Phenomena McGraw Hill Book Company. New York 1969, p270.

(37) Ranjan, M.; Numazawa, S.; Mukherjee, S. Local flux governing mechanism for the self-assembly of silver nanoparticles on ripple patterned templates. Materials Research Express 2014, 1, 015038.

(38) Jose-Yacaman, M.; Gutierrez-Wing, C.; Miki, M.; Yang, D.-Q.; Piyakis, K.; Sacher, E. Surface diffusion and coalescence of mobile metal nanoparticles. The Journal of Physical Chemistry B 2005, 109, 9703-9711.

(39) Cinti, R.; Chakraverty, B. The growth of silver films on silica in ultra-high vacuum: I. Experimental-Growth model. Surface Science 1972, 30, 109-124. 